\title{
In Absence of Absenteeism: Some Thoughts on Productivity Costs in Economic Evaluations in a Post-corona Era
}

\author{
Werner Brouwer $^{1} \cdot$ Samare Huls $^{1} \cdot$ Ayesha Sajjad $^{1} \cdot$ Tim Kanters $^{1,2} \cdot$ Leona Hakkaart-van Roijen $^{1} \cdot$ Job van Exel $^{1}$
}

Accepted: 16 November 2021 / Published online: 16 December 2021

(c) The Author(s), under exclusive licence to Springer Nature Switzerland AG 2021

\section{Introduction}

The ongoing coronavirus ('corona') crisis has impacted individuals and societies around the globe in an unprecedented fashion. Next to human suffering in terms of health and wellbeing, the impacts of both the disease and responses to the disease on healthcare systems, societal functioning, and the economy have been and remain profound. It is not unlikely that some of these impacts will have a lasting influence on societies. One of the areas for which this may be the case is that of paid work and employment. Next to direct losses of jobs and economic decline, the way in which paid work is performed has changed due to the pandemic and the measures to contain it, like lockdowns and social distancing. Working from home has become much more common in many sectors and countries [1]. This change was facilitated by digital communication tools that rapidly became part of everyday life (also for academics, like it or not) and meant adaptation to a new way of working for many involved. The productivity of people working from home, relative to that in a formal workplace, especially during lockdowns and with little time to adjust, is a relevant and interesting issue to study in its own right, as highlighted in, for instance, Huls et al. [2]. Such studies can also be relevant in investigating the costs and benefits of different policy interventions related to reducing infection rates during a pandemic.

The corona crisis has not only emphasized the relevance of productivity costs related to illness and interventions, but may also be relevant for the methods used in health economic evaluations [3]. Because the crisis may have a lasting

Werner Brouwer

brouwer@eshpm.eur.nl

1 Erasmus School of Health Policy and Management, Erasmus University Rotterdam, P.O. Box 1738, 3000 DR Rotterdam, The Netherlands

2 Institute for Medical Technology Assessment (iMTA), Rotterdam, The Netherlands effect on how individuals perform their paid and unpaid work (which may also become more intertwined), it seems relevant to rethink whether and how we capture productivity costs in economic evaluations adequately. Productivity costs can be defined as the costs associated with paid and unpaid production loss and replacement due to illness, disability, or death of productive persons [4]. In this editorial, we provide some thoughts on the identification, measurement, and valuation of productivity costs in economic evaluations in a post-corona era, with an emphasis on the issue of valuing production losses in the absence of conventional absenteeism from paid work.

\section{Perspective}

Some have argued against adopting a broad societal perspective in economic evaluations in the healthcare sector and advocated taking a narrower healthcare perspective in which only health benefits and costs falling on the healthcare budget are deemed relevant [5-8]. This would imply excluding productivity costs of patients [9]. If anything, the current pandemic highlights, almost in extremis, how large the impacts of health (risks) and interventions in the healthcare sector can be on society and the economy. Not informing (healthcare) decision-makers about these impacts, as a rule, seems hard to defend, and it is difficult to imagine how optimal decisions can be made while systematically ignoring substantial and real societal impacts [10]. The current pandemic may therefore emphasize the relevance of adopting a broader perspective in economic evaluations in the healthcare sector. This ensures that all costs and benefits of an intervention are included, regardless of where or on whom they may fall [11], and that decision-makers are fully informed about the health and welfare consequences of interventions. This would also allow informed and explicit consideration of the distributional consequences of decisions, which are also highly relevant in the context of fighting a 
pandemic [12]. Equity is not necessarily served by ignoring the broader impacts of interventions.

Note that adopting a broader societal perspective does not and should not preclude analysts from also looking at the impact of an illness or intervention on the healthcare system per se and, for instance, its health opportunity (or displacement) costs [7, 8]. Indeed, during this crisis, such displacement costs also have become more tangible and visible than perhaps ever before, for example, due to shortages of intensive care beds and postponed non-coronavirus disease (non-COVID) care [13]). Both perspectives are relevant $[8,14,15]$.

\section{Absenteeism}

As productivity costs are commonly identified in relation to absence from the workplace [16], available methods and instruments facilitating the inclusion of productivity costs in economic evaluations $[17,18]$ may need adjustment, especially in case of a more permanent shift to working from home.

Absenteeism is often equated with a complete loss of productivity. However, the conventional notion of 'absence from work', with its strong connotation with the workplace, may become less relevant, less meaningful, and less useful when people work substantial proportions of their time from home. If the location where one works and where one stays when ill and unable to work is one and the same, working from home results in the absence of 'absenteeism' in its traditional meaning.

This implies that current methods for identifying and measuring productivity costs in terms of 'absenteeism' may need to be revised. People who work from home while feeling ill may not register as being 'absent' nor associate themselves with 'being absent from work'. Hence, when confronted with common questions in productivity costs questionnaires, such as 'How many workdays were you absent from work during the past 2 weeks?', they may not answer in a way that allows valid estimations of productivity costs, potentially leading to measurement errors. Although people can and likely will still call in sick on workdaysthey cannot perform their (full) tasks due to illness-this may not be perceived as the same as being 'absent'. Current methods and instruments for measuring and valuing absenteeism may therefore need to be re-evaluated and potentially adjusted when used in the context of working from home.

\section{Sick Days}

Observing the number of officially registered 'sick days' or asking respondents whether they called in sick (and how many days) may be one way forward. An essential question in this context is when people would call in sick in case of working from home as compared to working in a formal workplace (ceteris paribus). It has been suggested that absenteeism will typically occur when people fall below a certain threshold of impairment, productivity, or quality of life [19]. This is also likely the case for calling in sick when working from home, although the threshold level may differ between working from home and physically going to a workplace (and contextual factors such as type of work, type of commute, and social security arrangements, etc.). Equating 'reported sick days' in the case of working from home with 'being absent' in the case of working in a formal workplace may therefore not be completely accurate. This would also imply that comparing to or extrapolating productivity costs from pre-corona studies may be inaccurate since the level of ill-health or productivity for calling in sick post-corona would have to be the same as for being absent from work pre-corona. Further research into these matters is required.

\section{Productivity is Not Dichotomous}

Assuming sick days to be associated with zero productivity and all other days with full productivity, ${ }^{1}$ as commonly done when calculating productivity costs related to absenteeism, is an oversimplification, illustrated with three situations:

1. People may be ill and less productive without registering sick days or absenteeism. They may do less work or produce lower quality work and hence incur productivity losses, but these would not be measured when only focusing on absenteeism or sick days.

2. As mentioned before, there is a certain threshold of impairment, productivity, or quality of life for absenteeism. In other words, before calling in sick or when returning to work after illness, there is a period when people are less productive but not sufficiently so to call in sick. These productivity losses would also not be captured when focusing on absenteeism or sick days.

3. People may call in sick but still perform some tasks (at their own pace and time). This means that even when

\footnotetext{
${ }^{1}$ Note that in using the friction cost method, the valuation of lost working days is based on value added by employees but corrected for the elasticity for labour time versus labour productivity, which is assumed to be 0.8 [20].
} 
people are absent or have called in sick, productivity may not be zero.

It has been highlighted before that reduced productivity without absenteeism and before or after absenteeism is common and that the associated productivity costs may be substantial [21, 22]. This will likely also be the case when shifting the focus to measuring 'sick days' and especially also in the context of working from home. Therefore, it seems necessary to measure productivity in a less dichotomous fashion.

\section{Methods Presently Used for Measuring Presenteeism}

An alternative is to measure productivity in paid work on a continuous scale, ranging from being able to do as much as usual when healthy to not being able to perform any work. Such scales have been developed and validated, both in relation to the quantity and the quality of work performed [18, 21]. Currently, such scales are typically used in the context of presenteeism (if at all), as both quality and quantity are normally assumed to be zero in the case of absenteeism. However, particularly in the context of working from home, such scales could be helpful for coming to more accurate estimations of productivity costs when ill, regardless of whether people have called in sick or not (i.e., in all three situations listed above).

Identification and measurement of productivity (losses), especially in the case of working from home, may therefore benefit from focusing less on the status of people (being absent or having called in sick) and more on the actual level of productivity. Methods developed in the context of measuring presenteeism may then be a promising way forward. This could, for instance, be done by asking how much paid work people could perform on certain days. A question like 'On how many workdays were you unable to perform any paid work' would then indicate the number of workdays entirely lost, which would be interpretable both in the case of working from home or in a formal workplace, or when these two are combined ('hybrid working'). Moreover, for the days not fully lost to work, in line with common presenteeism questions, people could be asked about the quantity and quality of the work they were able to do. Validating such measures for this purpose is, of course, important, as is a better understanding of compensation mechanisms and multiplier effects in the context of working from home more often. Moreover, it will be interesting to investigate, like in Huls et al. [2], the determinants of productivity at home, including family composition, working space, type of work, etc. Finally, if in the post-corona era presenteeism becomes more and absenteeism less prominent, this may also have consequences for valuation methods, for instance, whether and how replacement, as assumed in the friction cost method (e.g. [9, 17]), plays a role in working from home and presenteeism.

\section{Terminology}

What these thoughts perhaps most strongly highlight is that our current terminology does not fit well with a future in which working from home will probably be more common. Absenteeism and presenteeism invoke associations with a workplace that is separate from the location where one would be when ill, which is not necessarily the case when working from home. Rather than focusing on 'being there', the focus should be on 'being productive', and from being absent or not to how much work someone could perform. Arguably, this will result in a more general and accurate account of productivity costs than currently the case, but it does require more individual level data collection (or accurate prediction models [23]).

\section{Unpaid Work}

In the context of working from home more often, it seems relevant to make a few remarks on unpaid work, which remains often ignored in economic evaluations, but clearly is not without value to individuals and society. It has been argued before that unpaid work should be included more systematically in economic evaluations (also to counter an inequitable focus on paid work only and therefore misrepresenting the productivity in particular groups) [24]. When working from home, working hours may be less well delineated, productivity in both areas may be more correlated, and joint production may occur more often (e.g., performing paid work while keeping an eye on the children or a sick housemate). As paid and unpaid work become more intertwined and measuring productivity in paid work from home more common, the step towards including productivity in unpaid work in economic evaluations becomes smaller and more relevant. However, an integrated view on and measurement of productivity requires further work on measuring and valuing productivity in unpaid work [17, 24].

\section{Macro Methods}

A final observation is that commonly used methods and instruments to measure and value productivity costs in economic evaluations especially pertain to the individual level and situations in which diseases have a marginal impact on the healthcare system and society—notwithstanding their 
potentially huge impact on individuals and their families, of course. The current pandemic is characterized by a nonmarginal impact at the system level, with some economic sectors coming to a (near) complete standstill. Methods to quantify and value such macro-economic impacts are not part of typical economic evaluations in healthcare, but are highly relevant in contexts of a pandemic like corona. Developing and expanding methods for economic evaluations in the context of fighting disruptive outbreaks with a 'systemic' impact should remain high on our agenda.

\section{Concluding}

If the corona crisis results in structural changes to the location of work, from a formal workplace-more often-to home, a rethink of methods for capturing productivity costs in economic evaluations of health interventions will be required, potentially accounting for the increasing relation between paid and unpaid work. We may also need to reconsider our terminology in this context. 'Being there'-as reflected in terms like absenteeism and presenteeism-may need to shift to 'being productive', that is, irrespective of the location of work. In any case, post-corona, even in the absence of conventional absenteeism, productivity changes related to illness and treatments will still occur and remain highly relevant for research and policy.

\section{Declarations}

Conflict of interest None of the authors has conflicts of interest to declare, other than our general interest in this topic. All co-authors read and approved the final version of the manuscript. No funding was received for preparing this editorial.

Ethics Approval Not applicable.

Consent to Participate Not applicable.

Consent for Publication Not applicable.

Availability of Data and Material Not applicable.

Code Availability Not applicable.

Author Contributions Not applicable.

\section{References}

1. A Bick, A Blandin, K Mertens. Work from Home Before and after the COVID-19 Outbreak. CEPR Discussion Paper No. DP15000, Available at SSRN https://ssrn.com/abstract $=3650114$.

2. Huls SPI, Sajjad A, Kanters TA, et al. Productivity of working at home and time allocation between paid work, unpaid work and leisure activities during a pandemic. Pharmacoeconomics. 2021. https://doi.org/10.1007/s40273-021-01078-7.
3. Asuka Y, Briggs A, Garrison LP, et al. Principles of economic evaluation in a pandemic setting: an expert panel discussion on value assessment during the coronavirus disease 2019 pandemic. Pharmacoeconomics. 2021;39:1201-8.

4. Brouwer WBF, Koopmanschap MA, Rutten FFH. Productivity costs measurement through quality of life? A response to the recommendations of the Washington Panel. Health Econ. 1997;6:253-9.

5. Johannesson M. A note on the depreciation of the societal perspective in economic evaluation of health care. Health Policy. 1995;33(1):59-66.

6. Drummond M, Sculpher MJ, Torrance GW, O'Brien BJ, Stoddart GL. Methods for the economic evaluation of health care programmes. 3rd ed. Oxford: Oxford University Press; 2005.

7. K Claxton, S Walker, S Palmer, M Sculpher. Appropriate Perspectives for Health Care Decisions. CHE Research Paper 54. Centre for Health Economics, University of York, UK, 2010

8. Brouwer WBF, van Baal PHM, van Exel NJA, Versteegh MM. When is it too expensive? Cost-effectiveness thresholds and health care decision making. Eur J Health Econ. 2019;20(2):175-80.

9. Sculpher M. The role and estimation of productivity costs in economic evaluation. In: Drummond M, McGuire A, editors. Theory and practice of economic evaluation in health. Oxford: Oxford University Press; 2001.

10. Krol HM, Papenburg-de Jong J, Koopmanschap MA, Brouwer WBF. Do productivity costs matter? The impact of including productivity costs on the cost-effectiveness of interventions targeted at depressive disorders. Pharmacoeconomics. 2011;29(7):601-19.

11. Gold MR, Siegel JE, Russell LB, Weinstein MC. Cost-effectiveness in health and medicine. New York: Oxford University Press; 1996.

12. Brouwer WBF. The inclusion of spillover effects in economic evaluations: not an optional extra. Pharmacoeconomics. 2019;37(4):451-6.

13. Gravesteijn B, Krijkamp E, Busschbach J, et al. Minimizing population health loss in times of scarce surgical capacity during the coronavirus disease 2019 crisis and beyond: a modeling study. Value Health. 2021;24(5):648-57.

14. Brouwer WBF, van Exel NJA, Baltussen RMPM, Rutten FFH. A dollar is a dollar is a dollar-or is it? Value Health. 2006;9(5):341-7.

15. Sanders GD, Neumann PJ, Basu A, Brock DW, Feeny D, Krahn M, Kuntz KM, Meltzer DO, Owens DK, Prosser LA, Salomon JA, Sculpher MJ, Trikalinos TA, Russell LB, Siegel JE, Ganiats TG. Recommendations for conduct, methodological practices, and reporting of cost-effectiveness analyses. JAMA. 2016;316(10):1093-103.

16. Pritchard C, Sculpher M. productivity costs: principles and practice in economic evaluation. Monographs, Office of Health Economics, number 000464; 2000.

17. Krol HM, Brouwer WBF. How to include productivity costs in economic evaluations. Pharmacoeconomics. 2014;32(4):335-44.

18. Bouwmans C, Krol HM, Severens JL, Koopmanschap MA, Brouwer WBF, Hakkaart-van Roijen L. The iMTA Productivity Cost Questionnaire: a standardized instrument for measuring and valuing health related productivity losses. Value Health. 2015;18(6):753-8.

19. Brouwer WBF, Meerding WJ, Lamers L, Severens H. Productivity and health-related quality of life - an exploration. Pharmacoeconomics. 2005;23(3):209-18.

20. Koopmanschap MA, Rutten FF, van Ineveld BM, van Roijen L. The friction cost method for measuring indirect costs of disease. J Health Econ. 1995;14:171-89.

21. Brouwer WBF, Koopmanschap MA, Rutten FFH. Productivity costs without absence. Measurement validation and empirical evidence. Health Policy. 1999;48:13-27. 
22. Brouwer WBF, van Exel NJA, Koopmanschap MA, Rutten FFH. Productivity losses before and after absence: as important as common? Health Policy. 2002;61(2):173-87.

23. Krol HM, Stolk EA, Brouwer WBF. Predicting productivity losses through quality of life data. Eur J Health Econ. 2014;15(5):465-75.
24. Krol HM, Brouwer WBF. Unpaid work in economic evaluations. Soc Sci Med. 2015;144:127-37. 